\title{
A CCD Based Vertex Detector
}

\author{
Konstantin Stefanov \\ STFC Rutherford Appleton Laboratory \\ On behalf of the Linear Collider Flavour Identification (LCFI) Collaboration
}

* Introduction

* Vertex Detector R\&D at LCFI

- Column-Parallel CCDs

- In-situ Storage Image Sensors

- Low Mass Mechanical Support

* Conclusion 


\section{Introduction}

What is required for the vertex detector at ILC:

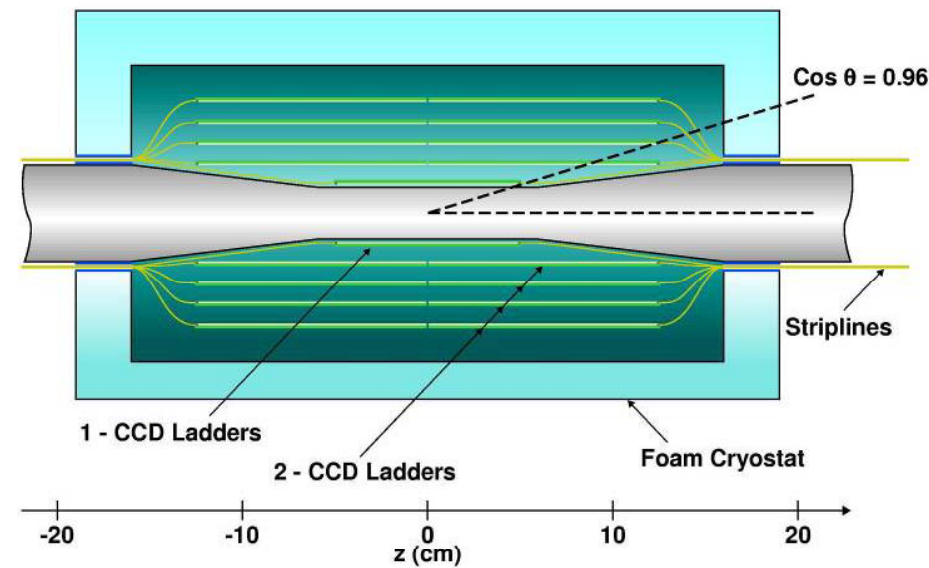

- Excellent point resolution ( $3.5 \mu \mathrm{m})$, small pixel size $=20 \mu \mathrm{m}$, close to IP

- Low mass ( $\leq 0.1 \% X_{0}$ per layer), low power dissipation

- Fast (low occupancy) readout - challenging, two main approaches

- Tolerates Electro-Magnetic Interference (EMI)

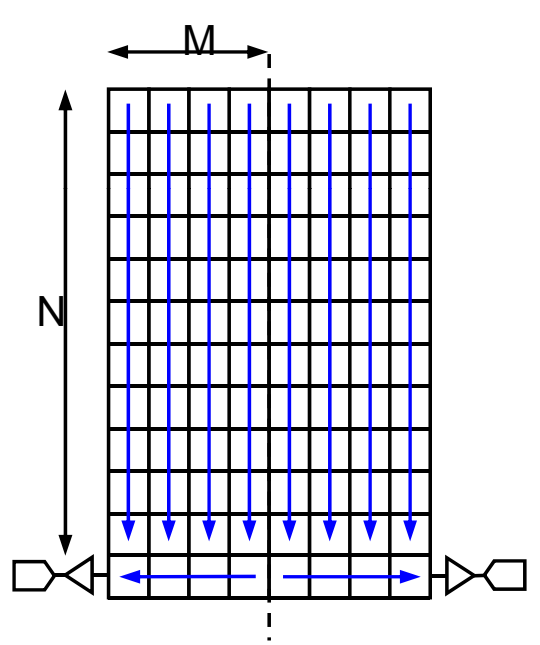

"Classic CCD"

Readout time $\approx$ $\mathrm{N} \times \mathrm{M} / \mathrm{f}_{\text {out }}$

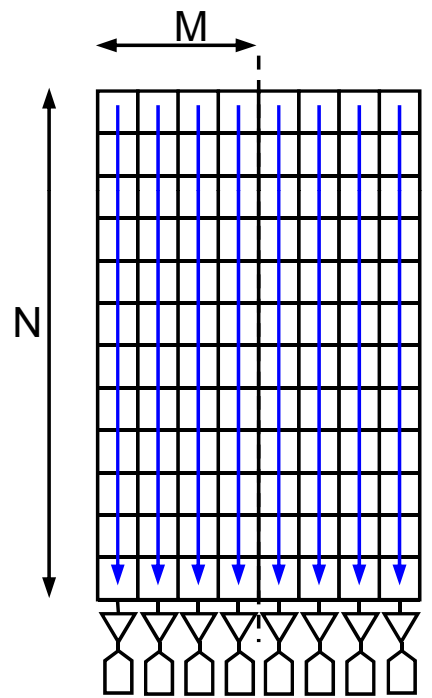

Column Parallel CCD

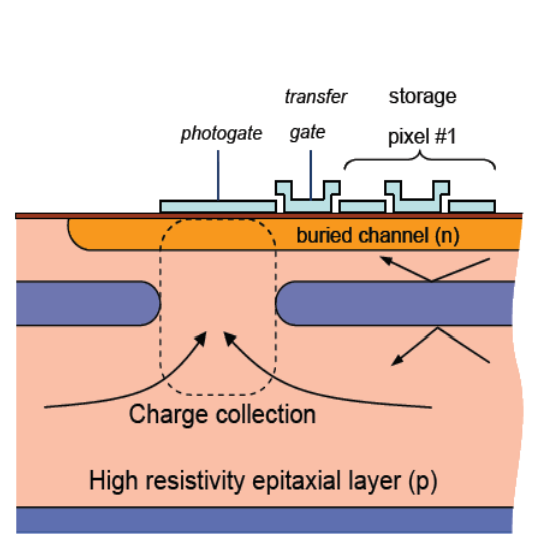

High resistivity epitaxial layer $(p)$

Readout time $=$ $\mathrm{N} / \mathrm{f}_{\text {out }}$

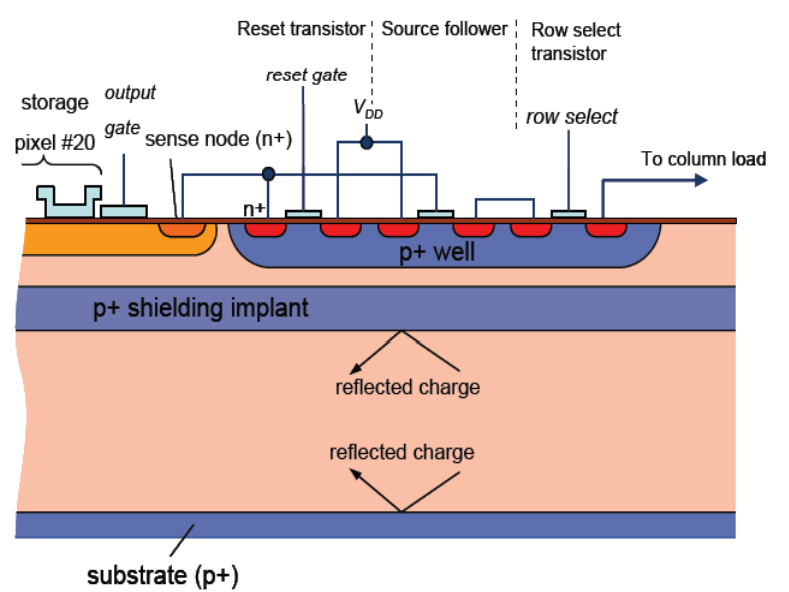

ISIS 


\section{CPCCD Programme: Bump-bonded CPC2/CPR2}

- Hybrid assembly CPC2/CPR2 fully tested

* Results incorporated into CPR2A

- Bump-bonded CPC2-40/CPR2 driven by two CPD1 chips

* Works up to $9 \mathrm{MHz}$

* $5 \mathrm{~cm}$ long CCD, signals pass 5 "stitches"

* Performance of the CPR2 is limiting factor, and gradually deteriorates at higher frequencies (missing and/or spurious codes)

* Sparse readout (below) up to $6 \mathrm{MHz}$

- Stand-alone CPC2/CPD1 reached $45 \mathrm{MHz}$ with transformer driver, $20 \mathrm{MHz}$ with CPD1 and trying to improve

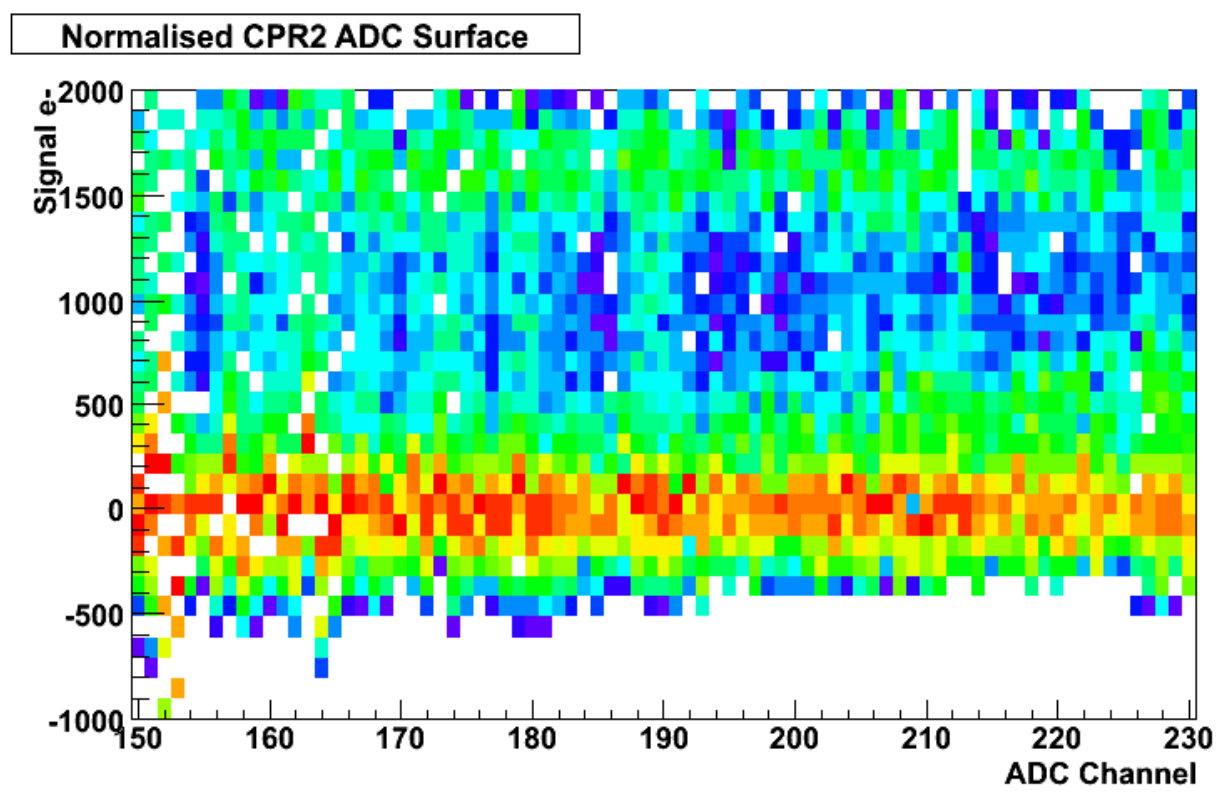

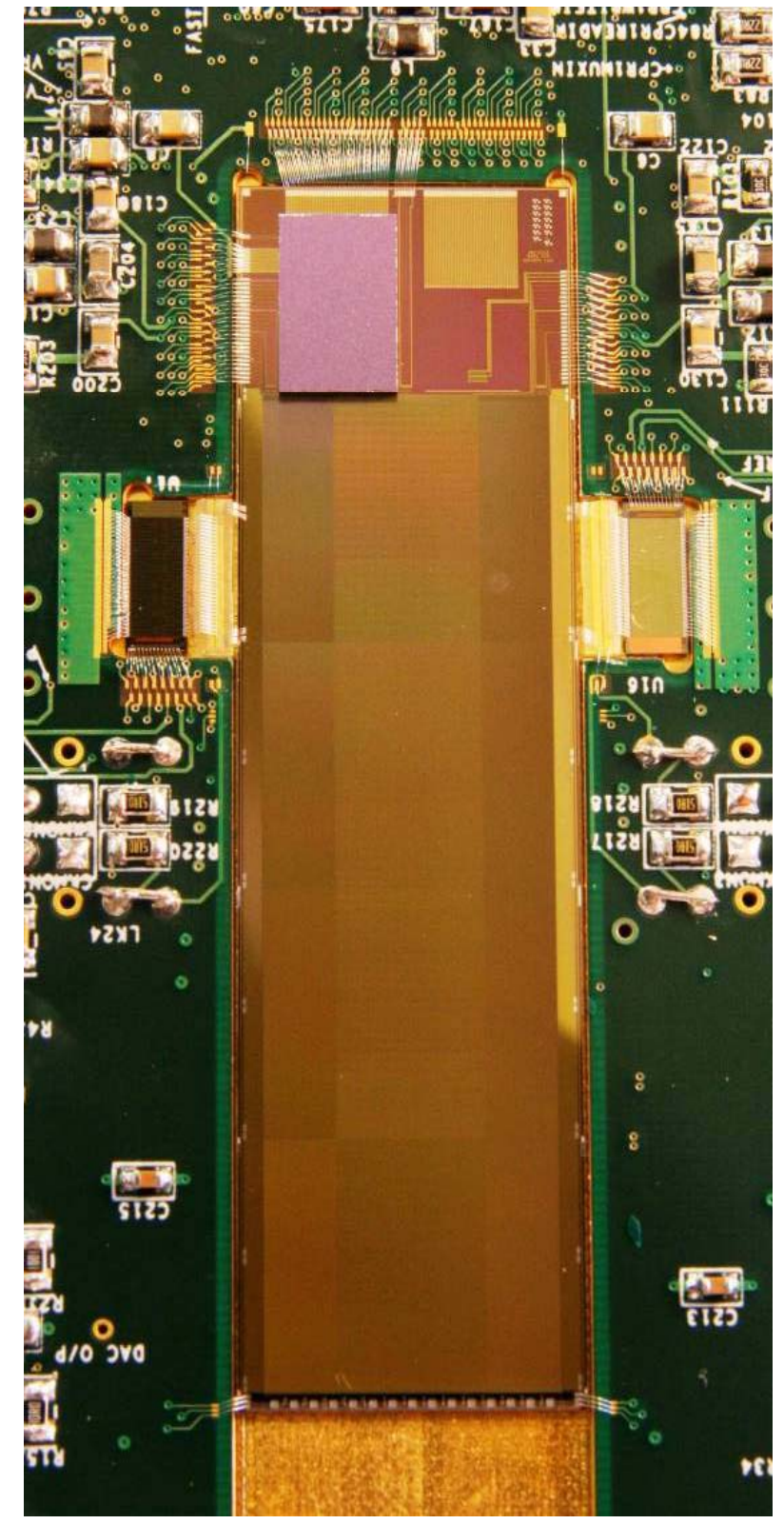

Tim Woolliscroft, Liverpool U 


\section{New Ideas: CCDs for Capacitance Reduction}
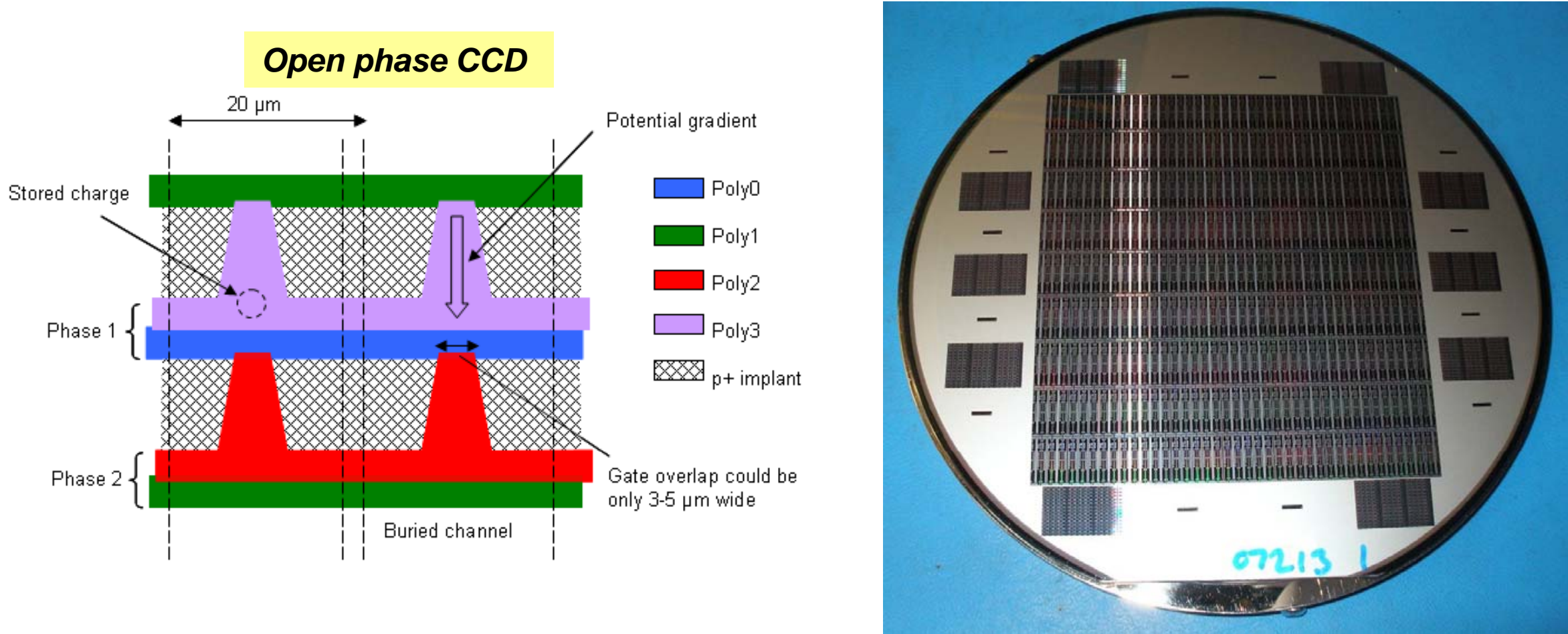

- High CCD capacitance is a challenge to drive because of the currents involved * New ideas to reduce the inter-gate capacitance $C_{\text {ig }}$ (by a factor 2-4) and clock amplitude

* Together with e2V Technologies designed 29 different types of small CCDs to test ideas

- 360 chips/wafer, first 6-inch wafers for LCFI 


\section{CPR2: Analogue Performance}

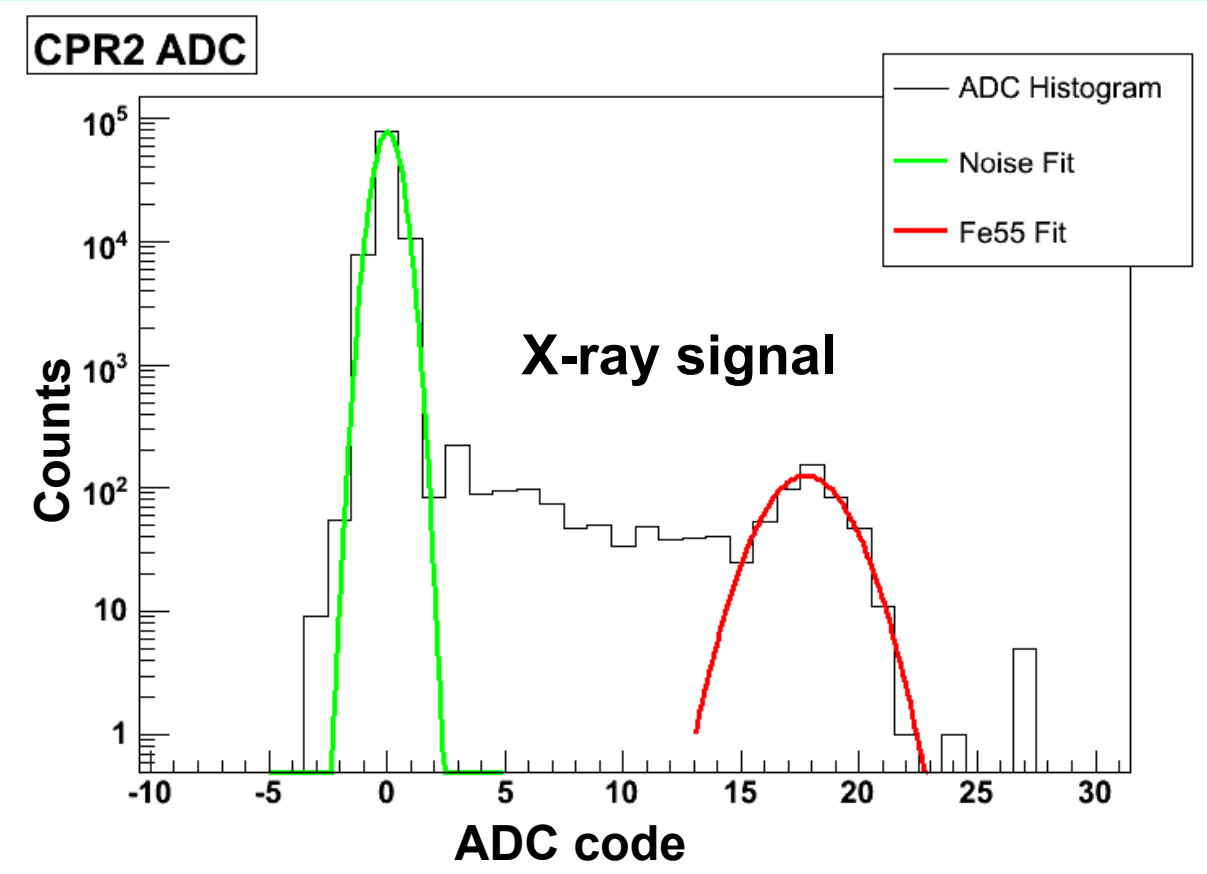

- Signals from all voltage channels observed (here at $2 \mathrm{MHz}, 300 \mathrm{mV}$ ADC range)

* Gain decreases by $50 \%$ away from the chip edges (as in CPR1)

- Noise around 60-80 e-

- Bump bond yield appears to be $100 \%$

- Charge channels did not work - swamped by digital crosstalk from the ADCs

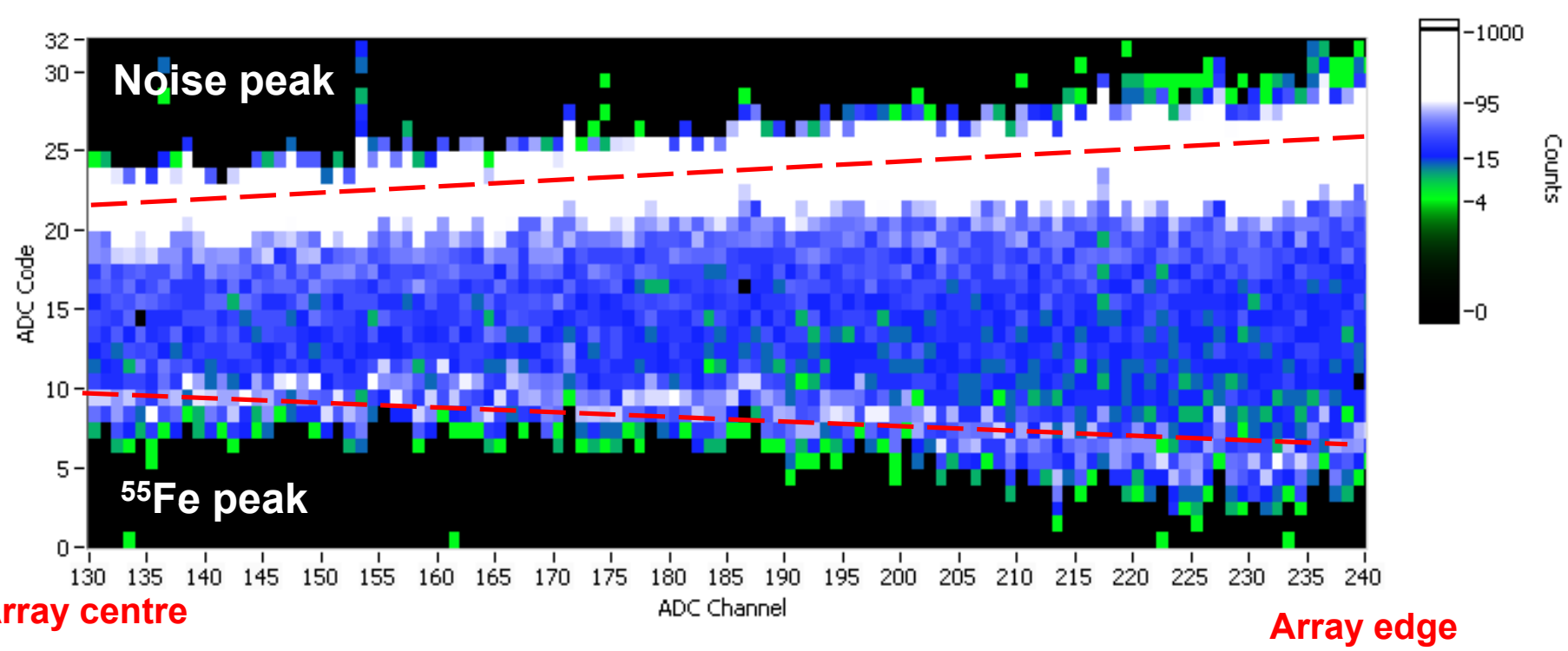




\section{The New Readout Chips CPR2A/B/C}

- Three variants: CPR2A, CPR2B and CPR2C submitted, delivery expected in mid-April

- The variants have different clock distribution - affects ADC performance;

- Expecting major improvements in functionality and performance:

* Fully re-designed cluster finder and sparsification circuitry - should cope better with high peak hit densities; added individual column threshold;

* Calibration circuit for both charge and voltage channels - allows analogue standalone testing of multiple channels;

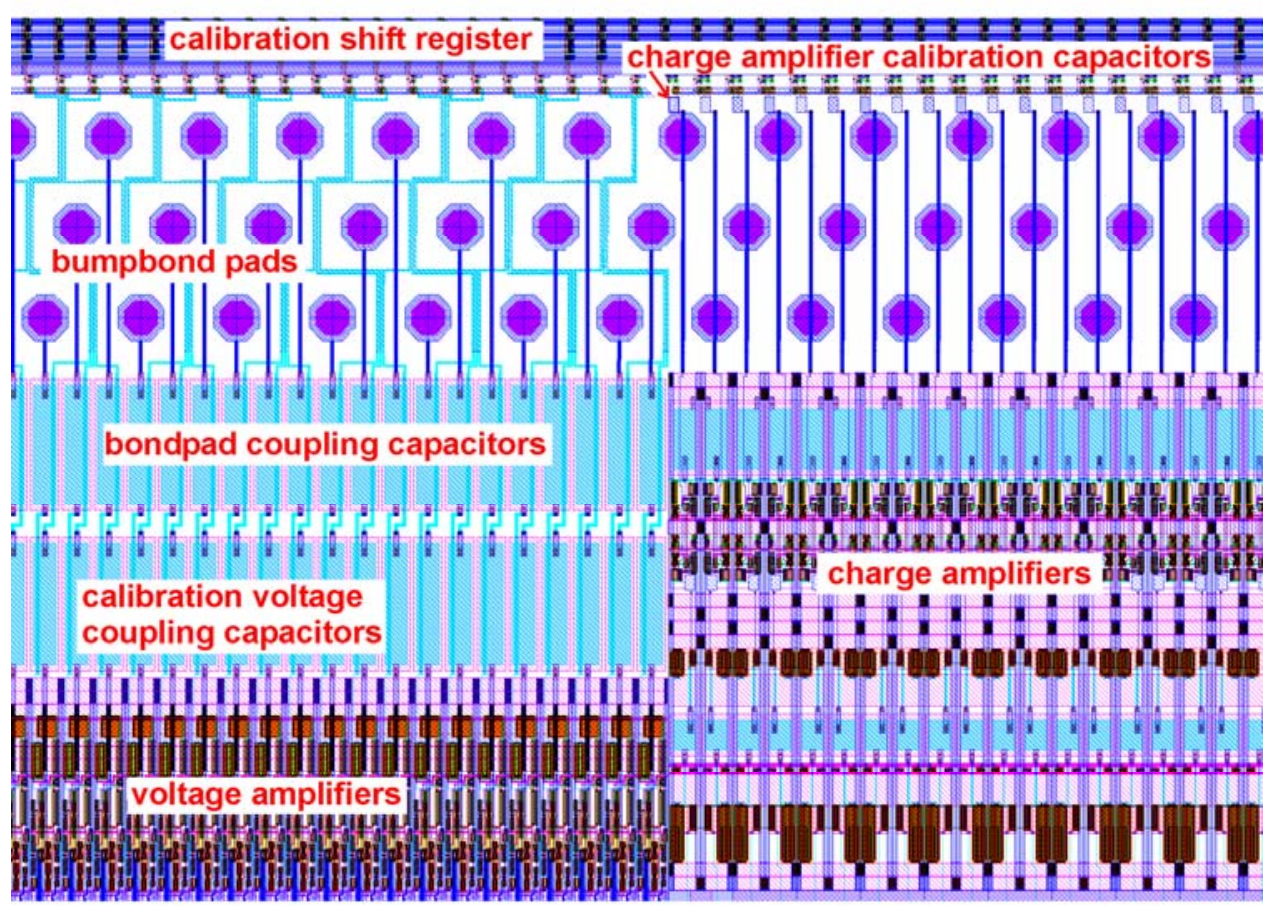

* Near-constant power supply current in the ADCs (externally controlled) - should reduce interference to the charge channels

* Gain matching between voltage channels to per cent level, achieved by lower track resistance and more stable current sources 


\section{Clock Driver for CPC2 : CPD1}

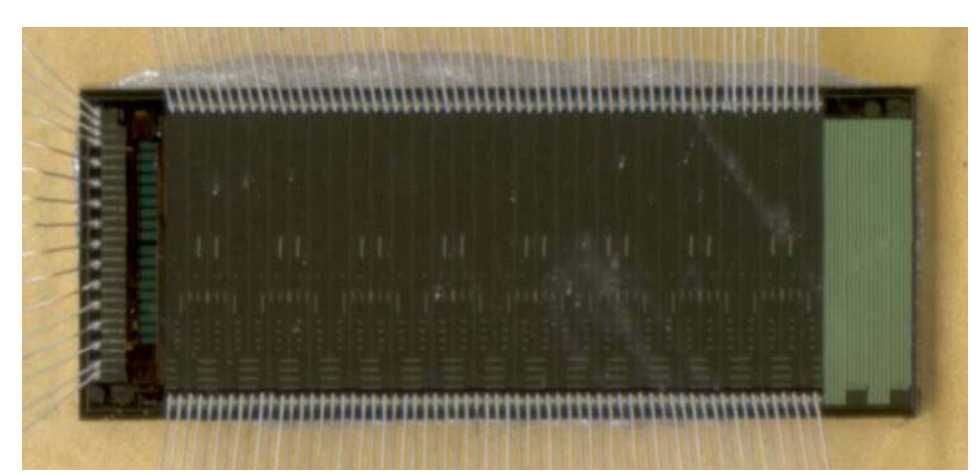

Steve Thomas, Peter Murray, RAL
- Designed to drive:

* Outer layer CCDs (127 nF/phase) at $25 \mathrm{MHz}$

* L1 CCD (40 nF/phase) at $50 \mathrm{MHz}$

* CPC2 requires 21 Amps/phase!

- One chip drives 2 phases, up to $3.3 \mathrm{~V}$ clock swing

- $0.35 \mu \mathrm{m}$ CMOS process, chip size $3 \times 8 \mathrm{~mm}^{2}$

- Careful layout on- and off-chip to cancel inductance

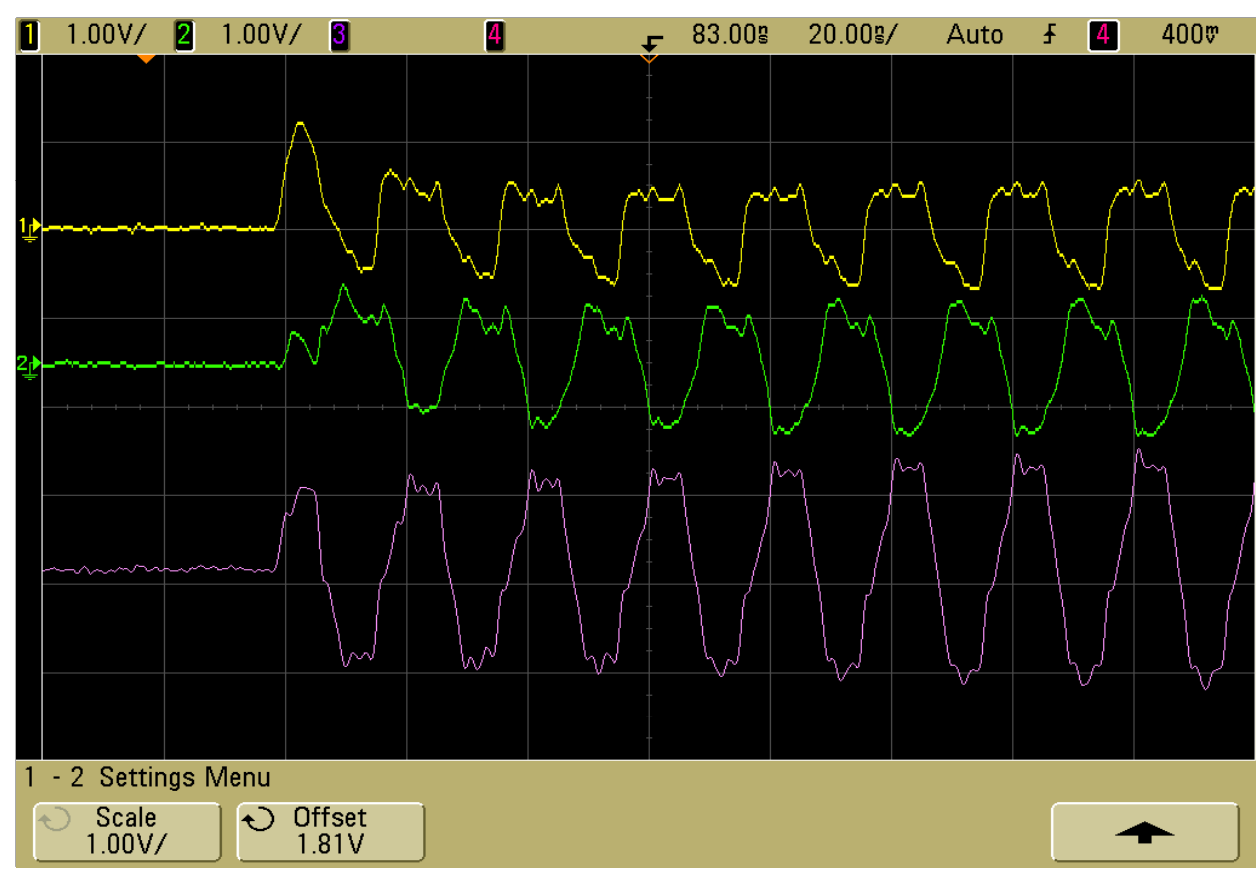

\section{Tests:}

- CPD1 driving $32 \mathrm{nF}$-equivalent internal load at $50 \mathrm{MHz}$

- Hope to maintain the same performance when bump-bonded to a CCD

$2 V_{p k-p k}$ differential clocks

Rui Gao, Andrei Nomerotski, Oxford U 


\section{In-situ Storage Image Sensor (ISIS)}
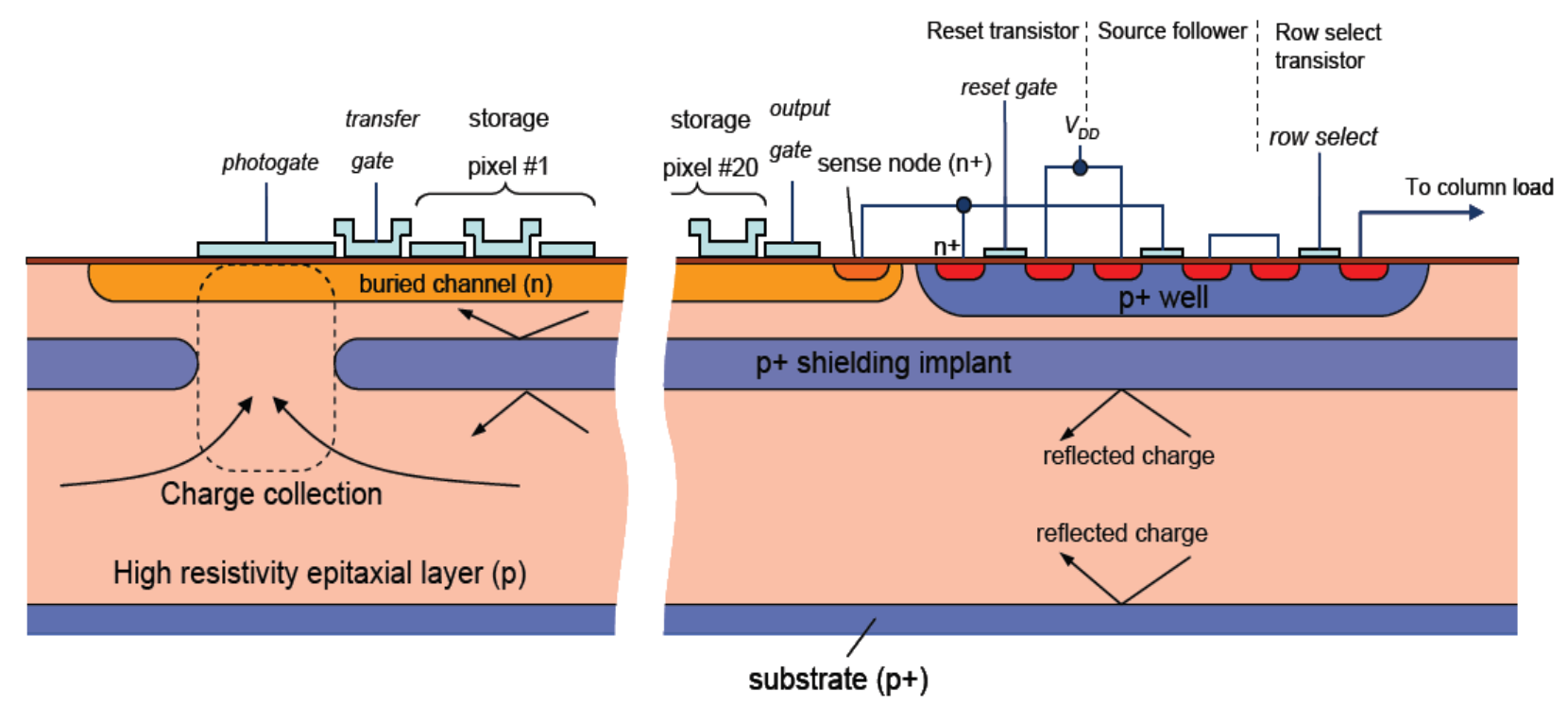

Chris Damerell, RAL

\section{Operating principles of the ISIS:}

1. Charge collected under a photogate;

2. Charge is transferred to 20-pixel storage CCD in situ, 20 times during the 1 ms-long train;

3. Conversion to voltage and readout in the $\mathbf{2 0 0} \mathrm{ms}$-long quiet period after the train (insensitive to beam-related RF pickup);

4. $1 \mathrm{MHz}$ column-parallel readout is sufficient; 


\section{ISIS1 (p-well) - Experimental Results}

- In the new, revised p-well ISIS1 we studied :

* Is the p-well reflecting charge as intended?

* When and where is the p-well punched through?

- Using $5.9 \mathrm{keV} X$-rays (30 $\mu \mathrm{m}$ attenuation length in $\mathrm{Si}$, converted charge in $\approx 1 \mu \mathrm{m}$ sphere)
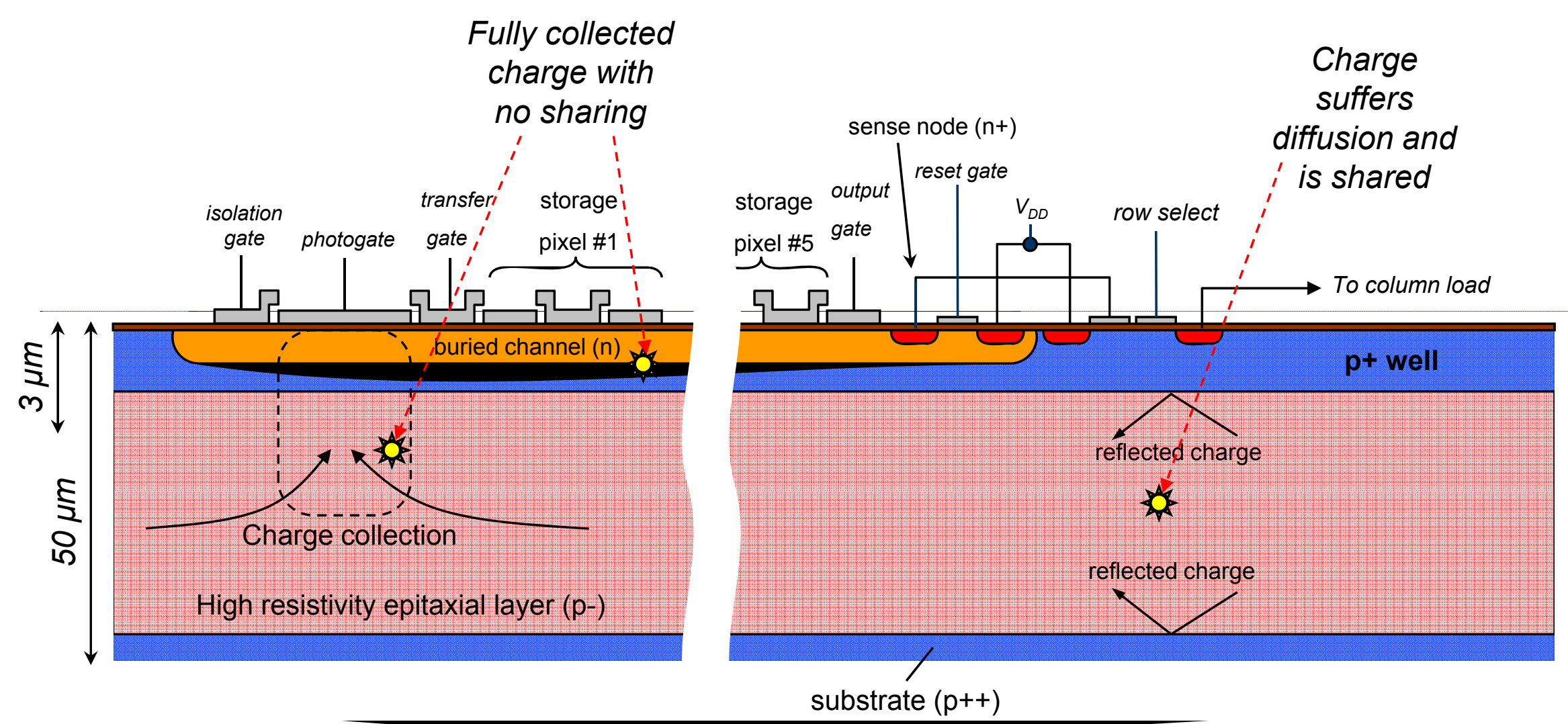


\section{ISIS1 (p-well)}

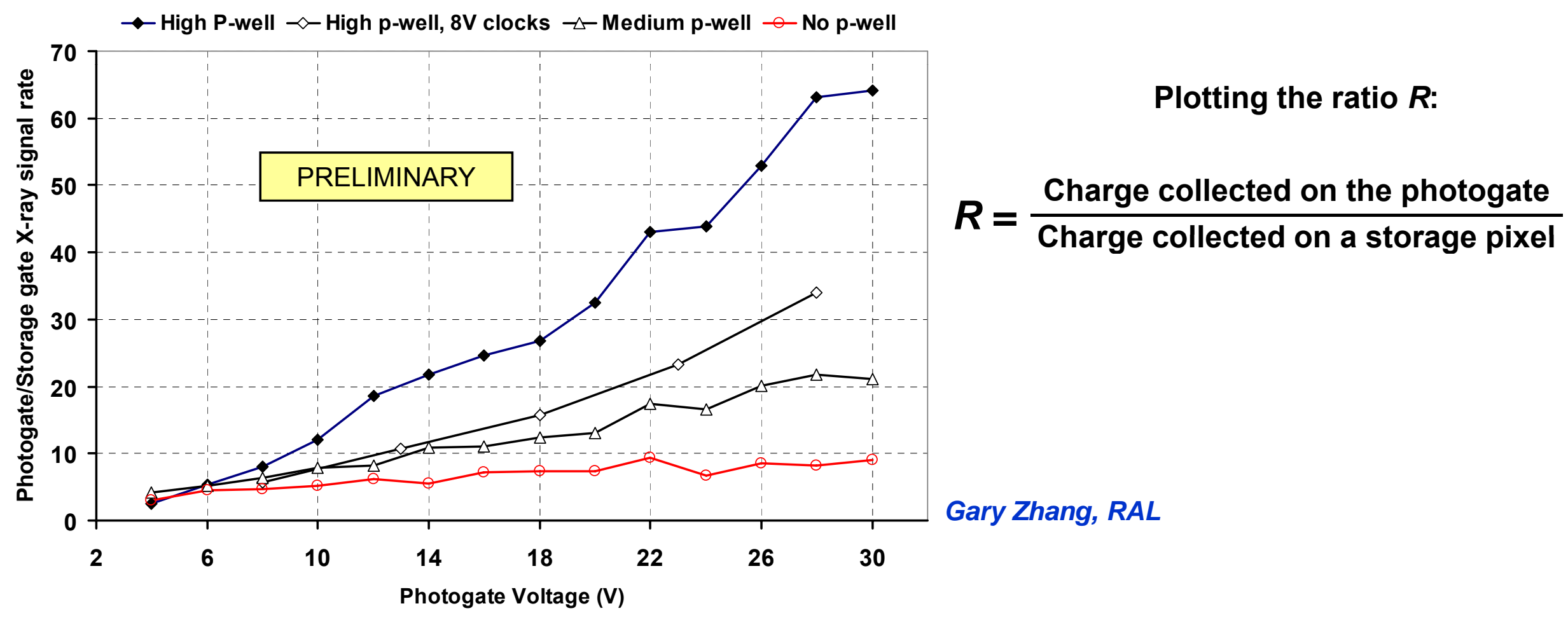

- "High p-well" - good protection of the storage register due to charge reflection

- The p-well works!

* The p-well under the storage gates can be punched through by higher clock amplitudes $-R$ drops

- Lower ratio with the medium-doped $p$-well due to lower potential barrier

- "No p-well" - only gate geometry and bias play role, $R$ is the lowest 


\section{Development of the New ISIS2}

- Jazz Semiconductor agreed to develop the CCD buried channel implant and the deep $p+$ implant

- We supply our custom doping profiles

- The process is $0.18 \mu \mathrm{m}$ dual gate oxide $(1.8 \mathrm{~V} / 5 \mathrm{~V})$

- Wafers with custom epitaxial layer: $25 \mu \mathrm{m}, \geq 100 \Omega$.cm on p++ substrate

- Will use $1 / 4$ of the mask set +2 additional masks on MPW to reduce costs

- Option to process 5 or 10 wafers, each with process variations, e.g. different doping levels

- Our wafers will be processed independently from the other customers'

- Area $=1 \mathrm{~cm}^{2}$

- Design is well advanced!

- $40 \%$ complete: pixel layout and logic for row selection done 


\section{ISIS2 - Chip Layout}

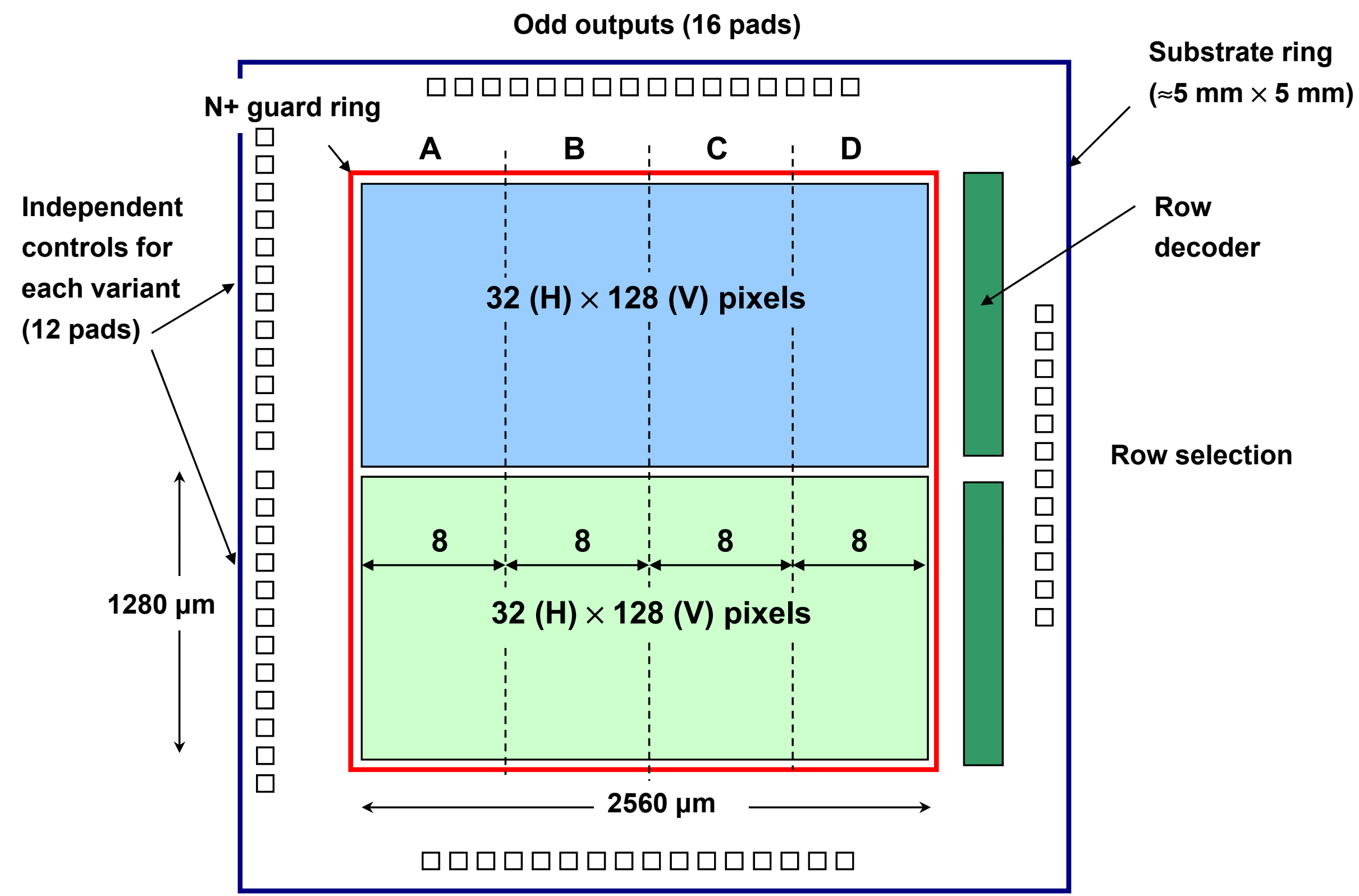

Even outputs (16 pads) 


\section{ISIS2 - Preliminary Pixel Layout}

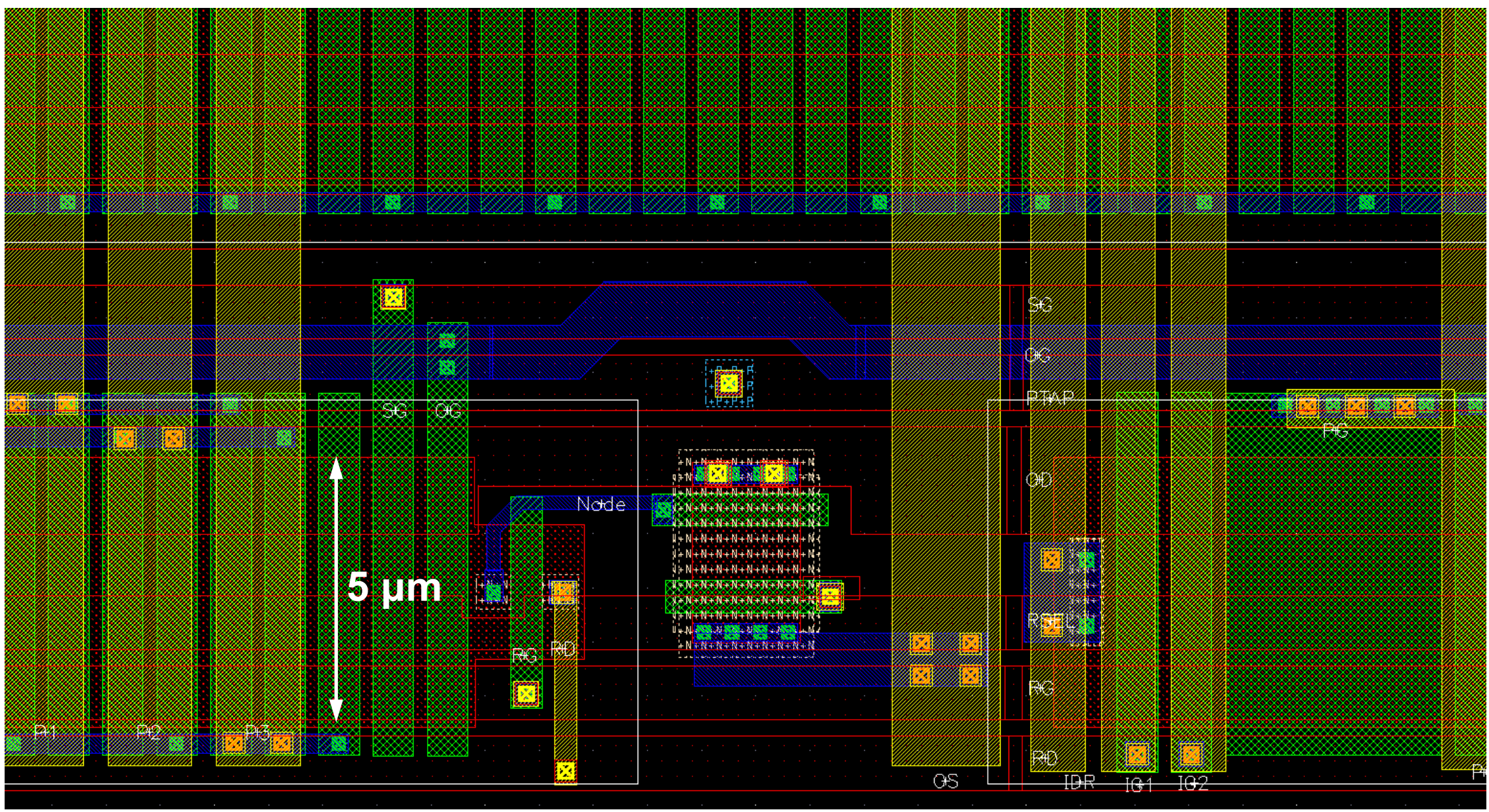

- $80 \mu \mathrm{m} \times 10 \mu \mathrm{m}$ pixels, $5 \mu \mathrm{m}$ wide buried channel, 3 metal layers

- Consulting with CCD and CMOS experts

- Target tape-out date $=8$ April 2008 


\section{Mechanical Support Studies}

- Goal is $0.1 \% X_{0}$ per ladder or better, while allowing low temperature operation ( 170 K)

- Active detector thickness is only $20 \mu \mathrm{m}$

- Unsupported silicon

- Stretched thin sensor $(50 \mu \mathrm{m})$, prone to lateral deformation

* Fragile, practically abandoned

- Silicon on thin substrates

- Sensor glued to semi-rigid substrate held under tension

- Thermal mismatch is an issue - causes the silicon to deform

- Many studies done for Be substrate

- Silicon on rigid substrates

- Shape maintained by the substrate

- Materials with good thermal properties available

- Foams offer low density and mass while maintaining strength 


\section{Mechanical Support Studies}

- RVC (Reticulated Vitreous Carbon) and silicon carbide are excellent thermal match to silicon

- Silicon-RVC foam sandwich ( 3\% density)

- Foam (1.5mm thick), sandwiched between two $25 \mu \mathrm{m}$ silicon pieces - required for rigidity

- Achieves $0.09 \% \mathrm{X}_{0}$

- Silicon on SiC foam ( 8\% density)

- Silicon $(25 \mu \mathrm{m})$ on SiC foam (1.5mm);

- Achieves $0.16 \% \mathrm{X}_{0}$

$\bullet 0.09 \% X_{0}$ possible with lower density foams (< $\left.5 \%\right)$

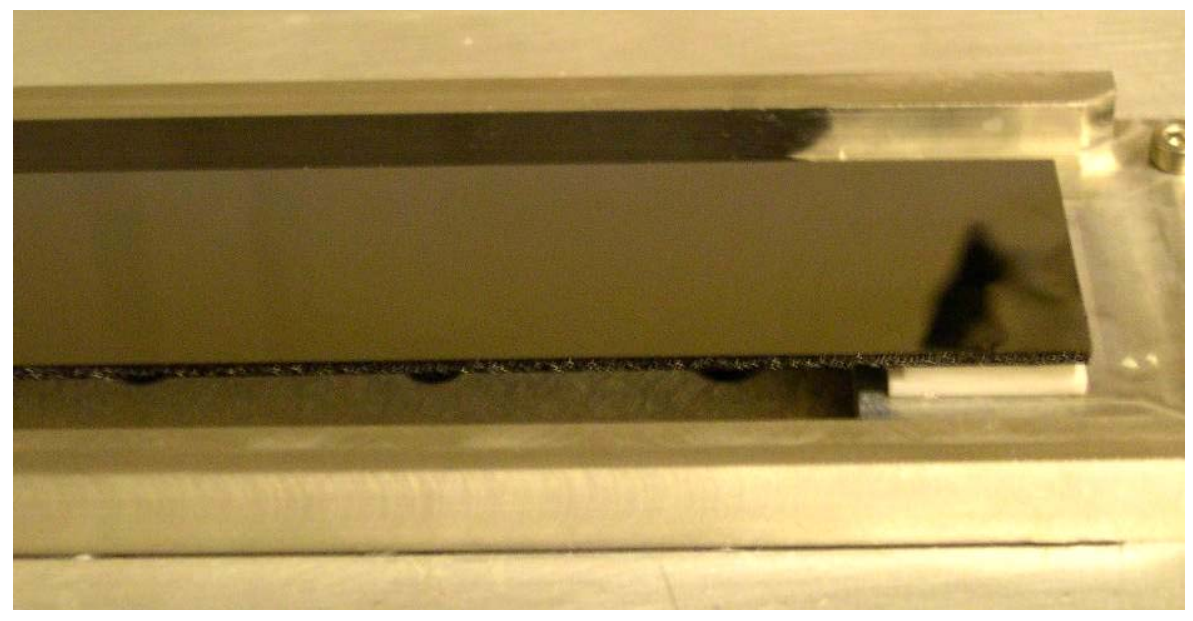

RVC foam (foam thickness $1.5 \mathrm{~mm}$ )

Thanks to Erik Johnson, RAL

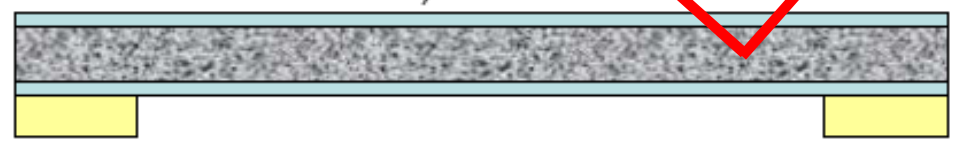

Silicon Carbide foam (foam thickness $1.5 \mathrm{~mm}$ )

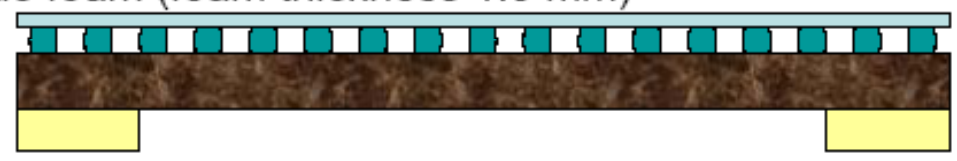




\section{Conclusion}

- Detector R\&D is progressing very well

- CPCCD programme:

* Bump-bonded assemblies CPC2-40/CPR2 reach 9 MHz

* Programme for capacitance and clock amplitude reduction underway

* Driver system with CMOS ASICs or transformers

* Third generation readout chip CPR2A to be delivered in April

- ISIS development:

- ISIS1 with p-well shows good protection from parasitic charge collection

- Now designing ISIS2 in CMOS process

- Mechanical support aims at $\leq 0.1 \% X_{0}$ using modern materials 


\section{Extra Slides}




\section{CPC1/CPR1 Performance}

$5.9 \mathrm{keV}$ X-ray hits, $1 \mathrm{MHz}$ column-parallel readout

Charge outputs, inverting (positive signals)

Noise $\approx 100$ e-

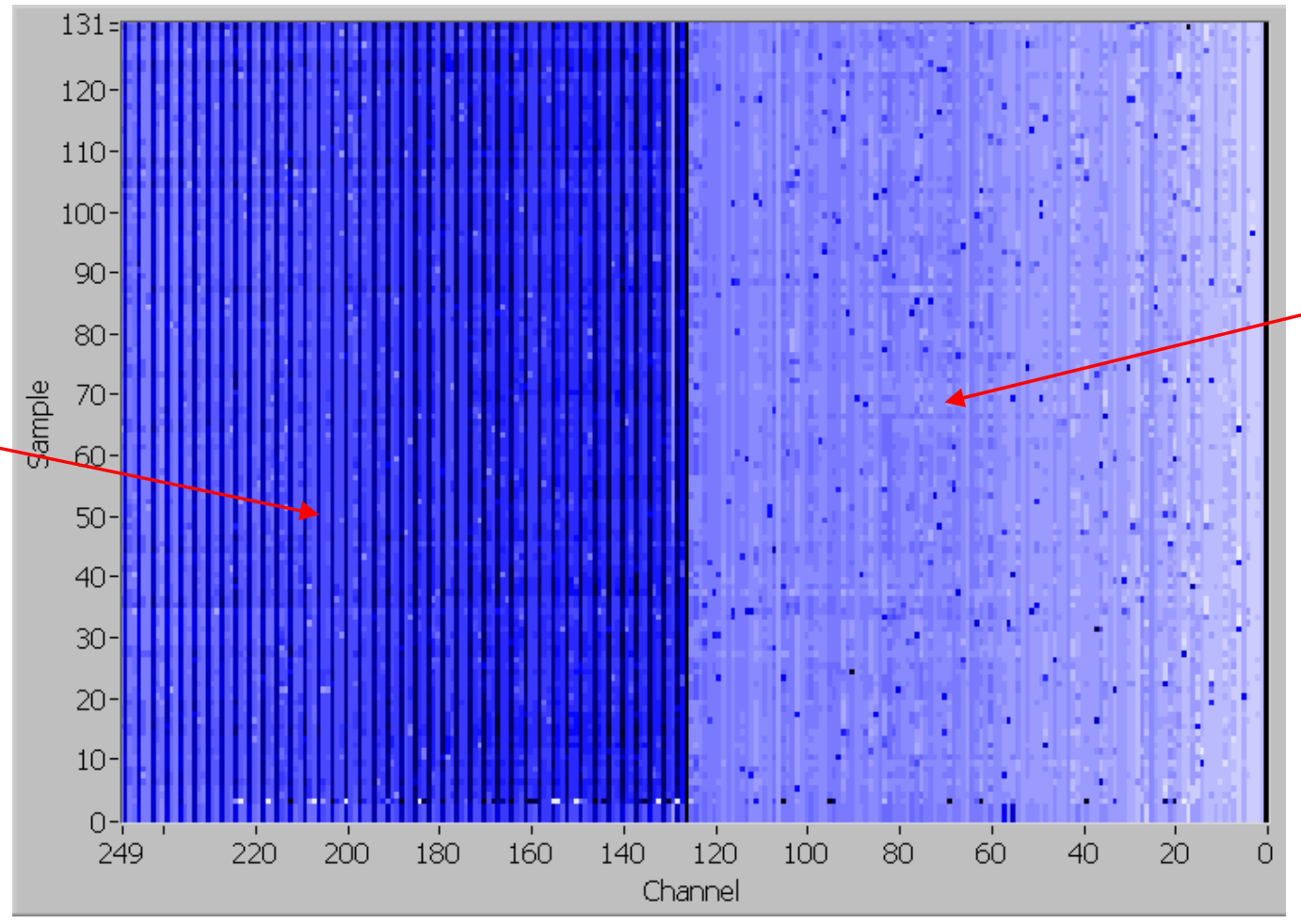

- First time e2V CCDs have been bump-bonded

- High quality bumps, but assembly yield only $30 \%$ : mechanical damage during compression suspected

- Differential non-linearity in ADCs (100 mV full scale) : addressed in CPR2
Voltage outputs, noninverting (negative signals)

Noise $\approx 60$ e-

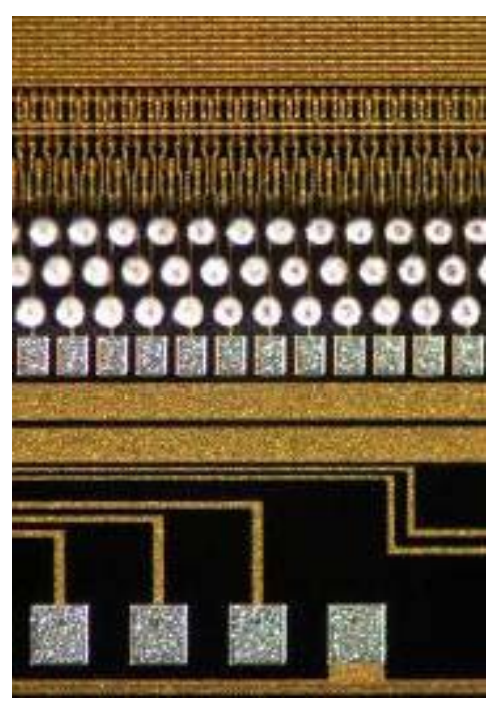

Bump bonds on CPC1 under microscope 


\section{The Column Parallel CCD}

- Main detector work at LCFI

- Every column has its own amplifier and ADC - requires readout chip

- Readout time shortened by orders of magnitude

- All of the image area clocked, complicated by the large gate capacitance

- Optimised for low voltage clocks to reduce power dissipation

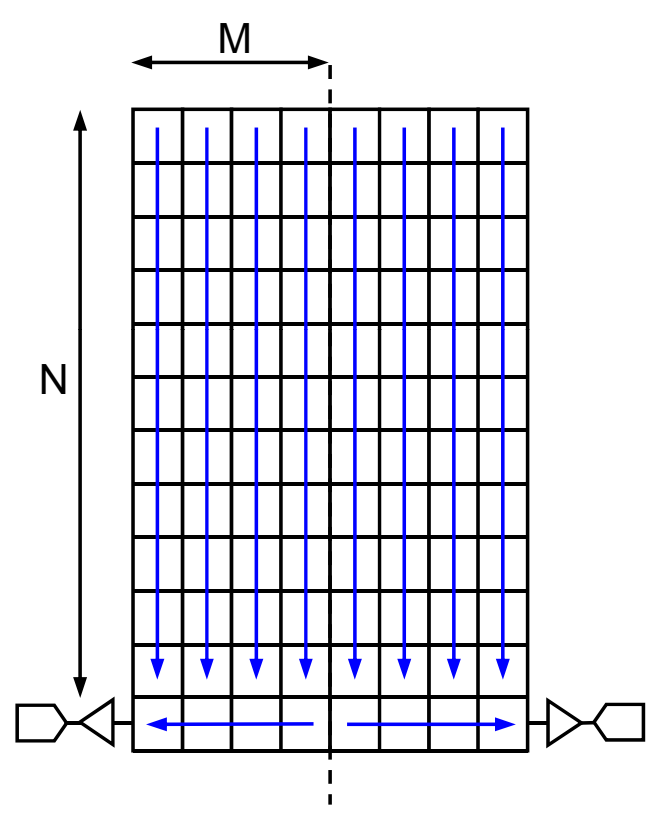

"Classic CCD"

Readout time $\approx$ $\mathrm{N} \times \mathrm{M} / \mathrm{f}_{\text {out }}$

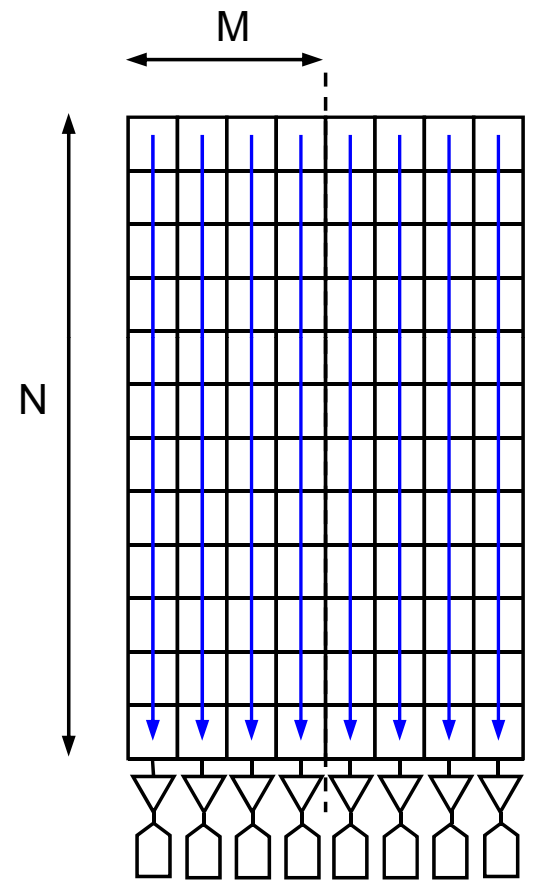

Column Parallel CCD

Readout time $=\mathrm{N} / \mathrm{f}_{\text {out }}$

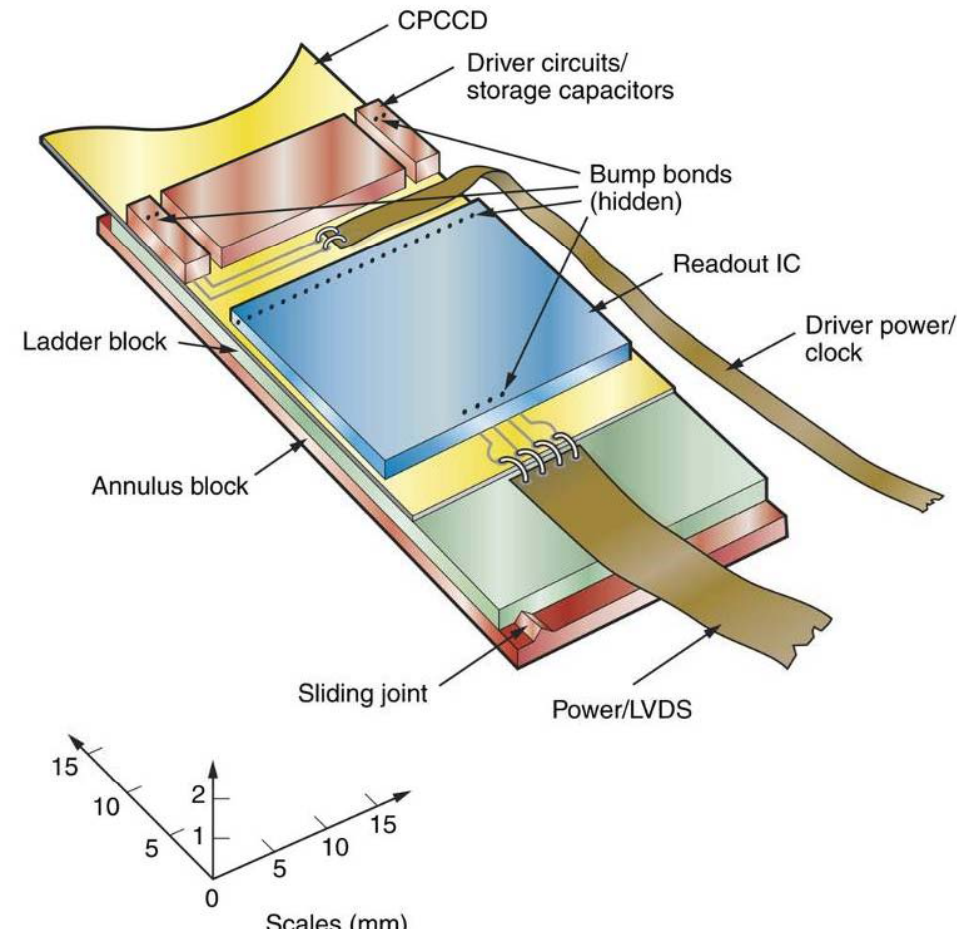

Scales $(\mathrm{mm})$ 


\section{CPC2, CPR2 and CPD1 All Together}

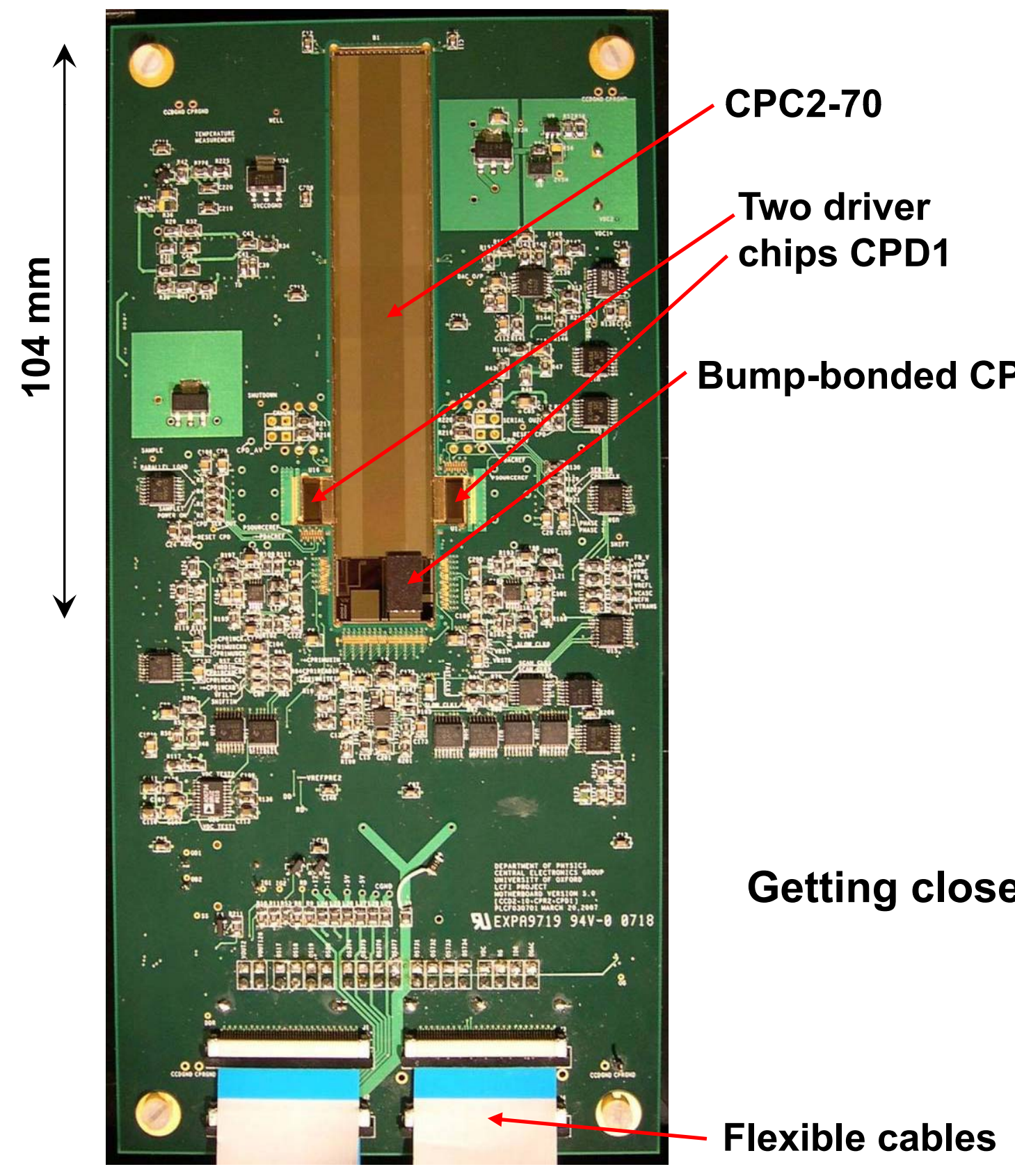

